\title{
An Adaptive Clarke Transform Based Estimator for the Frequency of Balanced and Unbalanced Three-Phase Power Systems
}

\author{
Elias Aboutanios \\ School of Electrical and \\ Telecommunications Engineering \\ University of New South Wales Australia \\ Email: elias@unsw.edu.au
}

\begin{abstract}
In this paper we examine the general problem of estimating the frequency of a balanced or unbalanced three-phase power system. The Clarke transform is commonly employed to transform the three real voltages to in-phase and quadrature components that are combined to form a complex exponential, the frequency of which can then be estimated. The imbalance between the voltages in an unbalanced system results in significant performance degradation. We address this problem by generalising the Clarke transformation to the case where the voltages are not equal. We then propose a new simple yet accurate algorithm for the estimation of the frequency. We simulate the algorithm and show that it achieves the performance that is obtained in the balanced case, practically sitting on the CramérRao Bound.
\end{abstract}

Index Terms-Three Phase Power Systems, frequency estimation, unbalanced power system, amplitude imbalance, Fast Iterative Interpolated DFT.

\section{INTRODUCTION}

The ability to control the system and maintain its stability depends on the accurate estimation of its frequency [1], [2]. This problem has become all the more important with the penetration of renewable energy sources and the advent of smart grids [3], [4]. In this work, we consider the general frequency estimation problem for a balanced or unbalanced three-phase system where the imbalance manifests as voltage sags [4].

The problem of estimating the frequency of a sinusoidal signal has been extensively researched [5], [6]. When the signal is complex, the frequency can be simply and efficiently estimated by many robust algorithms such as those in [6]. When multiple complex exponentials are present, however, the leakage effect must be accounted for [7]. Similarly, a real signal can be modeled as a sum of two complex exponentials which makes leakage compensation schemes necessary [3]. This approach has been applied in [8] to three phase systems where three filters were used to reduce these effects. More recently, a new real signal frequency estimator with built-in leakage compensation was proposed [9], [10]. This algorithm is powerful and effective for a single phase.

In a three-phase system, the well known Clarke Transform is usually used to obtain an in-phase and quadrature com- ponents that are then combined into a complex exponential. However, the Clarke Transform and the algorithms that employ it are applicable only to balanced systems. When the voltages deviate from the assumed model, as in the case of voltage sags [4], significant degradation in the performance can result. Previous efforts, such as those of [4], [11], [12], to tackle this problem have focused on deriving the signal model and modifying the frequency estimators to handle it. This leads to more complex estimators. In this work, we re-examine the transformation itself and generalise it to the unbalanced case. This allows us to employ simple frequency estimators directly giving a performance that sits on the Cramér-Rao Bound [6]. Therefore, we make two contributions. The first is the proposal and derivation of the adaptive Clarke transform (ACT). Although it is formulated here only for amplitude imbalance, this transformation has the potential to be extended to other forms of imbalance. The second contribution is the presentation of a novel estimation strategy that uses the ACT to achieve accurate frequency estimation under amplitude imbalance.

The rest of the paper is organised as follows. In Section II, we give the three-phase signal model and briefly review the Clarke Transform. The new, adaptive Clarke Transform (ACT) is presented in Section III. The ACT is then used in Section IV to propose a novel frequency estimation approach that can account for the unbalanced case. Section V reports simulation results to demonstrate the performance of the new approach. Finally, some conclusions are drawn in Section VI.

\section{The Clarke Transform}

Consider a three phase power system and assume we have $N$ samples for each of the $a, b$ and $c$ phases. The signal model is given by

$$
\begin{aligned}
& x_{a}[k]=v_{a}[k]+w_{a}[k] \\
& x_{b}[k]=v_{b}[k]+w_{b}[k] \\
& x_{c}[k]=v_{c}[k]+w_{c}[k],
\end{aligned}
$$


where the noise samples, $w_{a}, w_{b}$ and $w_{c}$, are assumed to be independent and identically distributed Gaussian with zero mean and variance $\sigma^{2} / 2$. The noiseless voltages are given by

$$
\begin{aligned}
& v_{a}[k]=V_{a} \cos (2 \pi f k+\phi) \\
& v_{b}[k]=V_{b} \cos \left(2 \pi f k+\phi-\frac{2 \pi}{3}\right) \\
& v_{c}[k]=V_{c} \cos \left(2 \pi f k+\phi+\frac{2 \pi}{3}\right) .
\end{aligned}
$$

Here $k=0 \ldots N-1, f$ is the system frequency, and $\phi$ the initial phase. The amplitudes of the three phases are denoted $V_{a}, V_{b}$ and $V_{c}$ respectively.

Instead of operating on each of the phases individually, the Clarke transform permits them to be combined into two orthogonal and one zero component, denoted by $\alpha, \beta$, and 0 . Since we are only interested in the complex exponential, we only use the $\alpha-\beta$ part of the Clarke transform, which we denote by $\mathbf{T}$. Thus,

$$
\mathbf{x}_{\alpha \beta}[k]=\mathbf{T} \mathbf{x}_{a b c}[k],
$$

where

$$
\mathbf{x}_{\alpha \beta}[k]=\left[\begin{array}{c}
x_{\alpha}[k] \\
x_{\beta}[k]
\end{array}\right], \text { and } \mathbf{x}_{a b c}[k]=\left[\begin{array}{c}
x_{a}[k] \\
x_{b}[k] \\
x_{c}[k]
\end{array}\right],
$$

and

$$
\mathbf{T}=\left[\begin{array}{ccc}
\frac{2}{3} & -\frac{1}{3} & -\frac{1}{3} \\
0 & \frac{1}{\sqrt{3}} & -\frac{1}{\sqrt{3}}
\end{array}\right] .
$$

Now, it is easy to verify that if $V_{a}=V_{b}=V_{c}=V$, then we have that $x_{\alpha}[k]=V \cos (2 \pi f k+\phi)$ and $x_{\beta}[k]=V \sin (2 \pi f k+\phi)$. This leads to

$$
\begin{aligned}
x[k] & =x_{\alpha}[k]+j x_{\beta}[k] \\
& =V e^{j 2 \pi f k+\phi}+w[k],
\end{aligned}
$$

where $w[k]$ is the resulting complex circular Gaussian noise component with variance $\frac{2 \sigma^{2}}{3}$. The combination of the three phase voltages results in an SNR gain of 1.5 times with respect to each individual phase, and the resulting complex exponential in white noise model simplifies many signal processing tasks including that of estimating the signal frequency.

\section{The Generalised Clarke Transformation}

When the system is unbalanced, the standard Clarke Transform does not produce a single exponential. While most approaches in the literature try to deal with the resulting more complicated signal model, we opt to re-examine the transformation itself to achieve a simplified signal model, specifically a signal model like (6). Thus, in what follows we will derive the general transformation for the unbalanced case where the phase voltages are not necessarily equal. Let the general transformation be

$$
\mathbf{G}=\left[\begin{array}{ccc}
\alpha_{a} & \alpha_{b} & \alpha_{c} \\
\beta_{a} & \beta_{b} & \beta_{c}
\end{array}\right] .
$$

Keeping in mind that the transformation is applied on a sample by sample basis, we ignore the time index for sake of notational simplicity. Applying $\mathbf{G}$ to the three phase voltages we get

$$
\begin{aligned}
v_{\alpha}= & \left(\alpha_{a} V_{a}-\frac{1}{2} \alpha_{b} V_{b}-\frac{1}{2} \alpha_{c} V_{c}\right) \cos (2 \pi k f+\phi) \\
& +\frac{\sqrt{3}}{2}\left(\alpha_{b} V_{b}-\alpha_{c} V_{c}\right) \sin (2 \pi k f+\phi), \text { and } \\
v_{\beta}= & \left(\beta_{a} V_{a}-\frac{1}{2} \beta_{b} V_{b}-\frac{1}{2} \beta_{c} V_{c}\right) \cos (2 \pi k f+\phi) \\
& +\frac{\sqrt{3}}{2}\left(\beta_{b} V_{b}-\beta_{c} V_{c}\right) \sin (2 \pi k f+\phi) .
\end{aligned}
$$

In order to obtain a complex exponential we require the coefficient of the cosine term in $v_{\alpha}$ to be equal to that of the sine term in $v_{\beta}$. Without loss of generality we set them equal to 1 . Furthermore, the coefficients of the sine term in $v_{\alpha}$ and of the cosine term in $v_{\beta}$ to be zero. Therefore,

$$
\begin{aligned}
\alpha_{a} V_{a}-\frac{1}{2} \alpha_{b} V_{b}-\frac{1}{2} \alpha_{c} V_{c} & =1 \\
\frac{\sqrt{3}}{2}\left(\alpha_{b} V_{b}-\alpha_{c} V_{c}\right) & =0 \\
\beta_{a} V_{a}-\frac{1}{2} \beta_{b} V_{b}-\frac{1}{2} \beta_{c} V_{c} & =0 \\
\frac{\sqrt{3}}{2}\left(\beta_{b} V_{b}-\beta_{c} V_{c}\right) & =1 .
\end{aligned}
$$

Solving this system of equations, leads to the parametrisation of the transformation on $\alpha$ and $\beta$ as

$$
\begin{aligned}
& \alpha_{a}=\frac{1}{V_{a}}\left(1+V_{b} \alpha\right), \alpha_{b}=\alpha \text { and } \alpha_{c}=\frac{V_{b}}{V_{c}} \alpha \\
& \beta_{a}=\frac{1}{V_{a}}\left(V_{b} \beta-\frac{1}{\sqrt{3}}\right), \beta_{b}=\beta \text { and } \beta_{c}=\frac{1}{V_{c}}\left(V_{b} \beta-\frac{2}{\sqrt{3}}\right) .
\end{aligned}
$$

While these equations give a single complex exponential, they do not constitute a unique solution. Thus, in order to determine the solution that gives the optimal transform, we minimise the output noise variance. Applying the transformation to the noise we have that

$$
w=\left(\alpha_{a} w_{a}+\alpha_{b} w_{b}+\alpha_{c} w_{c}\right)+j\left(\beta_{a} w_{a}+\beta_{b} w_{b}+\beta_{c} w_{c}\right) .
$$

The output noise is therefore zero-mean. Furthermore, using the independence of the noise samples, we can show that the variance is given by

$$
\begin{aligned}
\sigma_{\text {out }}^{2} & =\mathrm{E}\left[w w^{*}\right] \\
& =\left(\alpha_{a}^{2}+\alpha_{b}^{2}+\alpha_{c}^{2}+\beta_{a}^{2}+\beta_{b}^{2}+\beta_{c}^{2}\right) \frac{\sigma^{2}}{2} .
\end{aligned}
$$

In order to minimise $\sigma_{\text {out }}^{2}$, we differentiate it with respect to $\alpha$ and $\beta$ and equate to 0 . Substituting (9) into (11), and proceeding to differentiate and equate to 0 yields

$$
\begin{aligned}
& \frac{\partial}{\partial \alpha}\left\{\frac{1}{V_{a}^{2}}\left(1+V_{b} \alpha\right)^{2}+\alpha^{2}+\frac{V_{b}^{2}}{V_{c}^{2}} \alpha^{2}\right\}=0 \\
& \Rightarrow \quad \frac{1}{V_{a}^{2}}\left(2 V_{b}+2 V_{b}^{2} \alpha\right)+2 \alpha+2 \frac{V_{b}^{2}}{V_{c}^{2}} \alpha=0 .
\end{aligned}
$$


Solving for $\alpha$ and putting $V_{T}^{2}=V_{a}^{2} V_{b}^{2}+V_{a}^{2} V_{c}^{2}+V_{b}^{2} V_{c}^{2}$ we get

$$
\alpha=-\frac{V_{b} V_{c}^{2}}{V_{T}^{2}} \text {. }
$$

Finally, we obtain

$$
\alpha_{a}=\frac{V_{a}\left(V_{b}^{2}+V_{c}^{2}\right)}{V_{T}^{2}}, \alpha_{b}=-\frac{V_{b} V_{c}^{2}}{V_{T}^{2}} \text {, and } \alpha_{c}=-\frac{V_{b}^{2} V_{c}}{V_{T}^{2}} .
$$

Now turning our attention to the $\beta$-component, and following a similar procedure, we arrive at the solution

$$
\beta=\frac{V_{b}}{\sqrt{3}} \frac{2 V_{a}^{2}+V_{c}^{2}}{V_{T}^{2}}
$$

leading to

$$
\begin{aligned}
\beta_{a} & =\frac{V_{a}}{\sqrt{3}} \frac{\left(V_{b}^{2}-V_{c}^{2}\right)}{V_{T}^{2}}, \beta_{b}=\frac{V_{b}}{\sqrt{3}} \frac{2 V_{a}^{2}+V_{c}^{2}}{V_{T}^{2}}, \\
\text { and } \quad \beta_{c} & =-\frac{V_{c}}{\sqrt{3}} \frac{2 V_{a}^{2}+V_{b}^{2}}{V_{T}^{2}} .
\end{aligned}
$$

The transformation parameters derived above minimise the noise variance while maintaining the output signal amplitude at 1 . As a result, the SNR is maximised. Since the generalised transformation depends on the power system parameters, in this case the amplitudes, we refer to it as an "Adaptive Clarke Transform" or ACT. The ACT is then expressed by

$$
\mathbf{G}=\frac{1}{V_{T}^{2}}\left[\begin{array}{ccc}
V_{a}\left(V_{b}^{2}+V_{c}^{2}\right) & -V_{b} V_{c}^{2} & -V_{b}^{2} V_{c} \\
\frac{V_{a}}{\sqrt{3}}\left(V_{b}^{2}-V_{c}^{2}\right) & \frac{V_{b}}{\sqrt{3}}\left(2 V_{a}^{2}+V_{c}^{2}\right) & \frac{V_{c}}{\sqrt{3}}\left(2 V_{a}^{2}+V_{b}^{2}\right)
\end{array}\right] .
$$

Putting $V_{a}=V_{b}=V_{c}=1$ yields the Clarke transform shown in (5), which elucidates the particular transformation adopted by Clarke as it maximises the output SNR. The output SNR in the general case is given by

$$
\begin{aligned}
\rho & =\frac{1}{\sigma_{\text {out }}^{2}} \\
& =\frac{1}{\alpha_{a}^{2}+\alpha_{b}^{2}+\alpha_{c}^{2}+\beta_{a}^{2}+\beta_{b}^{2}+\beta_{c}^{2}} \frac{2}{\sigma^{2}} \\
& =\frac{3}{2 \sigma^{2}} \frac{V_{T}^{2}}{V_{a}^{2}+V_{b}^{2}+V_{c}^{2}} .
\end{aligned}
$$

Regardless of the individual line voltages, the transformation that employs $\mathbf{G}$ of (17) results in a pure exponential. Thus, instead of deriving estimators that are adapted to the unbalanced case, we adapt the transformation which then permits the use of pure tone frequency estimators.

\section{A Practical Frequency Estimation Algorithm}

The ACT derived in the previous section yields a pure exponential, simplifying the signal model and permitting the use of any pure tone frequency estimators, such as those of [5], [6] for the power system frequency estimation. This, however, requires that the amplitudes of the individual phases are known. In general, however, considering that unbalanced power systems might be experiencing voltage dips or rises, the voltage levels would likely not be available. Therefore, we present in this section a practical estimation strategy that combines the generalised transformation with the Fast iterative interpolated DFT (FIID) algorithm of [6], [13].

In the absence of information to the contrary, we begin by assuming that the system is balanced. The optimal transformation is then given by the traditional CT of (5). Under this assumption, we apply the CT and proceed, using the FIID algorithm, to obtain an estimate, $\hat{f}$, of the system frequency. Since the system may be unbalanced, the estimate $\hat{f}$ will be biased due to the presence of the secondary component, [12]. To address this, we propose to use the estimate $\hat{f}$ to derive estimates of the voltage amplitudes that will be used in the ACT. This process is iterated until convergence. The estimates of the amplitudes are obtained using the well-known Maximum Likelihood (ML) estimator. Specifically, it is readily shown that for phase $p$, where $p=a, b$ or $c$, the ML estimator of the amplitude is given by

$$
\hat{V}_{p}=\left\|\boldsymbol{\Sigma}^{-1} \mathbf{Y}_{p}(\hat{f})\right\|,
$$

where $\|\mathbf{v}\|$ is the norm of the vector $\mathbf{v}$. The matrix $\boldsymbol{\Sigma}$ is symmetric, given by

$$
\boldsymbol{\Sigma}=\left[\begin{array}{ll}
\Sigma_{11} & \Sigma_{12} \\
\Sigma_{21} & \Sigma_{22}
\end{array}\right]
$$

with

$$
\begin{aligned}
& \boldsymbol{\Sigma}_{11}=\sum_{k=0}^{N-1} \cos ^{2}(2 \pi k \hat{f}), \\
& \Sigma_{22}=\sum_{k=0}^{N-1} \sin ^{2}(2 \pi k \hat{f}),
\end{aligned}
$$

and

$$
\boldsymbol{\Sigma}_{12}=\boldsymbol{\Sigma}_{21}=\sum_{k=0}^{N-1} \cos (2 \pi k \hat{f}) \sin (2 \pi k \hat{f})
$$

The vector $\mathbf{Y}_{p}(\hat{f})$ is given by

$$
\mathbf{Y}_{p}(\hat{f})=\left[\begin{array}{c}
\sum_{k=0}^{N-1} x_{p}[k] \cos (2 \pi k \hat{f}) \\
\sum_{k=0}^{N-1} x_{p}[k] \sin (2 \pi k \hat{f})
\end{array}\right]
$$

These amplitudes are then inserted into (17) to give the adapted CT. The algorithm is summarised in Table I. In the table, the matrix $\mathbf{X}_{a b c}$ is a $3 \times N$ matrix whose rows comprise the time samples of each phase. Similarly the matrix $\mathbf{X}_{\alpha \beta}$ contains the $N$ samples of the ACT output for the $\alpha$ and $\beta$ phases.

\section{Simulations}

The proposed algorithm employs the ACT in order to take full advantage of the excellent performance of the FIID algorithm. The resulting ACT-FIID algorithm should then emulate the FIID and exhibit an RMSE that is very close to the Cramèr-Rao Bound (CRB). In this section we verify this and compare our method to the LS-SDFT algorithm of [12]. This algorithm takes a sliding window of DFT coefficients and obtains an estimate of the frequency by accounting for the additional component resulting from the imbalance. Although 
TABLE I

The Proposed ACT-FIID Algorithm

\begin{tabular}{ll}
\hline \hline Put & $\mathbf{G}=\mathbf{T}$ (that is start with the CT) \\
Do & For $q=1$ to $Q$, loop: \\
& (i) $\mathbf{X}_{\alpha \beta}=\mathbf{G} \mathbf{X}_{a b c}$ \\
& (ii) $\mathbf{x}=\mathbf{X}_{\alpha \beta}(1,:)+j \mathbf{X}_{\alpha \beta}(2,:)$ \\
& (iii) $\hat{f}=\operatorname{FID}(\mathbf{x})$ \\
& (iv) Calculate $\mathbf{Y}_{p}(\hat{f})$ and $\boldsymbol{\Sigma}$ using (24) and (20) \\
(iv) $\hat{V}_{p}=\left\|\boldsymbol{\Sigma}^{-1} \mathbf{Y}_{p}(\hat{f})\right\|$, for $p \in\{a, b, c\}$ \\
(v) Update $\mathbf{G}$ according to (17) \\
\hline \hline
\end{tabular}

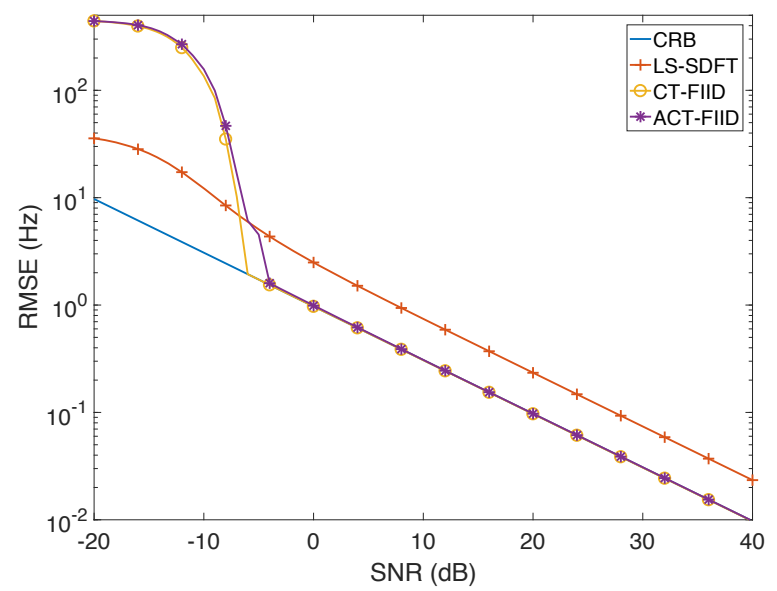

Fig. 1. Frequency RMSE vs SNR for the balanced case. 5000 MC runs were used.

[12] assumes that the sampling frequency is a multiple of the nominal frequency, LS-SDFT in fact is not critically dependent on this asumption. Finally we note that in the following simulations, we average 5000 Monte Carlo (MC) runs for each scenario.

\section{A. The Balanced Case}

Since the algorithm generalises the Clarke Transform, it should be able to achieve the same performance as the CT in the balanced case. To demonstrate this, we set the nominal frequency to $50 \mathrm{~Hz}$. For each $\mathrm{MC}$ run, we generate the actual frequency randomly in the interval $[49,51]$ and draw the phase from a uniform distribution over the interval $[-\pi, \pi]$. We simulate the estimation performance versus signal to noise ratio. For comparison, we include the CRB value in addition to the LS-SDFT, CT-FIID and proposed ACT-FIID. The Root Mean Square (RMSE) of the frequency in $\mathrm{Hz}$ is shown as a function of SNR in Fig. 1. To generate this figure, we used a value of $N=65$. For LS-SDFT we employed a DFT window of length 48 giving $L=16$ DFT coefficients. It is clear from Fig. 1 that LS-SDFT performs significantly worse than the other two methods. The CT-FIID method gives the ideal signal model in the balanced case and, therefore, achieves the CRB. Finally, we note that, as expected, ACT-FIID achieves the same performance as the CT-FIID. The minor difference around the breakdown threshold is attributed to the adaptation step, which is not required in the balanced case.

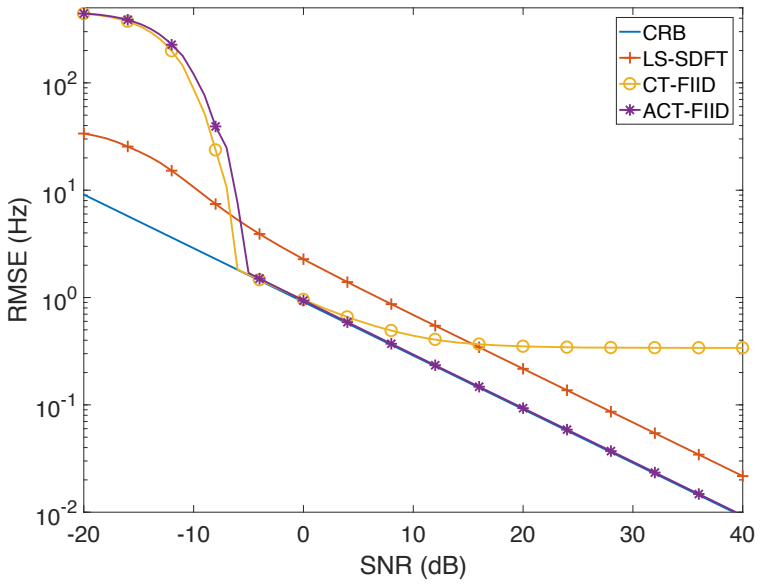

Fig. 2. Frequency RMSE vs SNR for the unbalanced case. 5000 MC runs were used.

\section{B. The Unbalanced Case}

Having shown that the proposed approach has desirable properties in the balanced case, we now look at its behaviour when the power system is unbalanced. To this end, we choose the same imbalance that was reported for the real system in [12]. That is we set $V_{a}=1.584, V_{b}=0.416$ and $V_{c}=1.305$. We generate the frequency and phase in exactly the same way as the previous example and keep the same parameters used for the algorithms. The results are shown in Fig. 2 below. We can clearly see that the ACT-FIID performs extremely well and achieves the CRB. As expected, the CT-FIID algorithm is heavily biased and deviates significantly from the CRB. It is interesting to note that the LS-SDFT shows a very similar performance as the previous example. This seems to indicate that the algorithm is able to deal with the system imbalance, but that the frequency estimation itself does not achieve the CRB.

\section{Conclusion}

The Clarke Transform (CT) has been become a ubiquitous tool for the analysis of power systems. However, the CT is not intended to map the three phases to signals that can be combined to form a pure tone. Rather it is meant to facilitate the analysis of the power system by transforming the three phases to two orthogonal axes and a dc term. Thus, while the CT has proven useful from an analysis point of view, it only has desirable properties in the balanced case. In this work we re-examined the CT from the signal processing perspective. We generalised it, under amplitude imbalance, with the goal of producing a pure tone with the maximum signal to noise ratio. We then employed this adaptive transformation to obtain a simple frequency estimation strategy that can achieve the CRB. The adaptive Clarke transform (ACT) has the potential to be extended to other forms of imbalance. Both the ACT and estimators based on it will be further investigated in future work. 


\section{REFERENCES}

[1] T. Lobos and J. Rezmer, "Real-time determination of power system frequency," IEEE Trans. on Instrum. and Meas., vol. 46, no. 4, pp. 877 -881 , aug. 1997.

[2] M. H. El-Shafey and M. M. Mansour, "Application of a new frequency estimation technique to power systems," IEEE Trans. on Power Del., vol. 21, no. 3, pp. 1045-1053, July 2006.

[3] T. Radil, P.M. Ramos, and A.C. Serra, "New Spectrum Leakage Correction Algorithm for Frequency Estimation of Power System Signals," IEEE Trans. on Instrum. and Meas., vol. 58, no. 5, pp. $1670-1679$, may. 2009.

[4] Y. Xia, S.C. Douglas, and D.P. Mandic, "Adaptive frequency estimation in smart grid applications: Exploiting noncircularity and widely linear adaptive estimators," IEEE Signal Process. Mag., vol. 29, no. 5, pp. 44-54, 2012.

[5] E. Aboutanios and B. Mulgrew, "Iterative frequency estimation by interpolation on fourier coefficients," IEEE Trans. on Signal Process., vol. 53, no. 4, pp. 1237-1242, April 2005.

[6] E. Aboutanios, "Estimating the parameters of sinusoids and decaying sinusoids in noise," IEEE Instrum. and Meas. Mag., vol. 14, no. 2, pp. 8-14, 2011.

[7] S. Ye and E. Aboutanios, "Rapid accurate frequency estimation of multiple resolved exponentials in noise," Signal Processing, vol. 132, pp. $29-39,2017$.

[8] J.K. Hwang and Y. Liu, "Noise analysis of power system frequency estimated from angle difference of discrete fourier transform coefficient," IEEE Trans. on Power Del., vol. 29, no. 4, pp. 1533-1541, 2014.

[9] S. Ye, D. L. Kocherry, and E. Aboutanios, "A novel algorithm for the estimation of the parameters of a real sinusoid in noise," in 2015 23rd European Signal Processing Conference (EUSIPCO), Aug 2015, pp. 2271-2275.

[10] S. Ye, J. Sun, and E. Aboutanios, "On the estimation of the parameters of a real sinusoid in noise," IEEE Signal Process. Lett., vol. 24, no. 5, pp. 638-642, 2017.

[11] T. Routtenberg and L. Tong, "Joint frequency and phasor estimation in unbalanced three-phase power systems," 2014, pp. 2982-2986.

[12] Y. Xia, K. Wang, W. Pei, Z. Blazic, and D. P. Mandic, "A least squares enhanced smart dft technique for frequency estimation of unbalanced three-phase power systems," in 2016 International Joint Conference on Neural Networks (IJCNN), July 2016, pp. 2762-2766.

[13] E. Aboutanios, "Generalised DFT-based estimators of the frequency of a complex exponential in noise," in Proceedings - 2010 3rd International Congress on Image and Signal Processing, CISP 2010, Yantai, 2010, vol. 6, pp. 2998-3002. 